\title{
Symmetry and Spin Dephasing in (110)-Grown Quantum Wells
}

\author{
V. V. Bel'kov, ${ }^{1,2}$ P. Olbrich, ${ }^{1}$ S. A. Tarasenko, ${ }^{2}$ D. Schuh, ${ }^{1}$ W. Wegscheider, ${ }^{1}$ T. Korn,,${ }^{1}$ C. Schüller, ${ }^{1}$ D. Weiss, ${ }^{1}$ \\ W. Prettl, ${ }^{1}$ and S. D. Ganichev ${ }^{1}$ \\ ${ }^{1}$ Terahertz Center, University of Regensburg, 93040 Regensburg, Germany \\ ${ }^{2}$ A. F. Ioffe Physico-Technical Institute, Russian Academy of Sciences, 194021 St. Petersburg, Russia
}

(Received 10 December 2007; published 2 May 2008)

\begin{abstract}
Symmetry and spin dephasing in (110)-grown GaAs quantum wells (QWs) are investigated applying magnetic field induced photogalvanic effect and time-resolved Kerr rotation. We show that magnetic field induced photogalvanic effect provides a tool to probe the symmetry of (110)-grown quantum wells. The photocurrent is only observed for asymmetric structures but vanishes for symmetric QWs. Applying Kerr rotation we prove that in the latter case the spin relaxation time is maximal; therefore, these structures set the upper limit of spin dephasing in GaAs QWs. We also demonstrate that structure inversion asymmetry can be controllably tuned to zero by variation of $\delta$-doping layer positions.
\end{abstract}

PACS numbers: 73.21.Fg, 72.25.Fe, 73.63.Hs, 78.67.De

Quantum wells (QWs) on (110)-oriented GaAs substrates have attracted growing attention in spintronics due to their extraordinary slow spin dephasing [1-4]. The reason for the long spin lifetime of several nanoseconds is the (110) crystal orientation: Then the effective magnetic field due to spin-orbit coupling points into the growth direction [5] and spins oriented along this direction do not precess. Hence the D'yakonov-Perel' spin relaxation mechanism [6], which is based on the spin precession in the effective magnetic field and usually limits the spin lifetime of conduction electrons, is suppressed. If, however, QWs are asymmetric, the structure inversion symmetry is broken and Rashba spin-orbit coupling causes an inplane effective magnetic field, thus speeding up spin dephasing. To judge the symmetry of QWs, one has to rely on the growth process, but no independent method to check the structure symmetry is readily available. Here we show that the magnetophotogalvanic effect (MPGE) [7] is an ideal tool to probe the symmetry of (110)-grown QWs. The photocurrent is only observed for asymmetric structures but vanishes if QWs are symmetric. We further show via time-resolved Kerr rotation that the spin relaxation time is maximal whenever the QW is symmetric.

Absorption of light in a quantum well in the presence of a magnetic field can generate a photocurrent. This is called as magnetophotogalvanic effect $[8,9]$. The microscopic model of the MPGE in QWs having bulk inversion asymmetry (BIA) and/or structure inversion asymmetry (SIA) is based on the asymmetry of photoexcitation and relaxation [10]. So far it has been observed in (001)-grown GaAs, SiGe, and InAs QWs [7,10,11]. The experiments here are carried out on molecular-beam epitaxy (110)-grown $\mathrm{Si}-\delta$-doped $\quad n$-type $\quad \mathrm{GaAs} / \mathrm{Al}_{0.3} \mathrm{Ga}_{0.7} \mathrm{As} \quad$ structures. Mobilities $\mu$ and carrier density $n_{s}$ are given in Table I. The conduction band profile of the investigated structures together with the corresponding $\delta$-doping position is shown in Fig. 1. QWs differ essentially in their doping profile: Sample A is a single heterojunction and has the strongest asymmetry stemming from the triangular confinement potential. In samples B and D, the doping layers are asymmetrically shifted off the barrier center either to the left or to the right, respectively (see Fig. 1). This asymmetric doping yields an asymmetric potential profile inside the QWs. To describe the degree of asymmetry we introduce the parameter $\chi=(l-r) /(l+r)$, where $l$ and $r$ are the spacer layer thickness between QW and the $\delta$-doping position (see Fig. 1 and Table I). Samples C and $\mathrm{E}$ contain a $\mathrm{Si}-\delta$ sheet, placed in the center of each barrier between adjacent QWs. While sample $\mathrm{E}$ was grown fully symmetrical, a weak asymmetry was added to sample C. There, the $\delta$ doping between first and second QWs was placed asymmetrically [see sample C in Fig. 1] thus introducing an asymmetry to the first quantum well. Samples grown along $z \|[110]$ were square shaped with the sample edges of $5 \mathrm{~mm}$ length oriented along $x \|[1 \overline{1} 0]$ and $y \|[00 \overline{1}]$. To measure photocurrents, Ohmic contacts were alloyed in the center of each sample side.

Photocurrents are measured at room temperature by exciting the samples with linearly polarized terahertz $(\mathrm{THz})$ radiation under normal incidence. The pulsed $\mathrm{THz}$ radiation is obtained from an optically pumped pulsed $\mathrm{NH}_{3}$ molecular laser [8]. The wavelength of $148 \mu \mathrm{m}$ was chosen to cause only intrasubband (Drude-like) absorption of the radiation. The geometry of the experiment is sketched in the inset of Fig. 2. The photocurrent is measured in unbiased structures via the voltage drop across a $50 \Omega$ load resistor. The width of the photocurrent pulses is about $100 \mathrm{~ns}$, which corresponds to the terahertz laser pulse duration. In the experiments described below we probe the sample asymmetry by means of the magnetic field dependence of the photocurrent with the polarization vector of the incoming light aligned along the $x$ axis. An external magnetic field with a maximum strength up to $B=0.5 \mathrm{~T}$ is applied either in plane, parallel to $y$, or normal to the $\mathrm{QW}$ plane. To relate the photocurrent and hence the asymmetry with spin lifetimes $\tau_{s}$ of the same devices, time-resolved 
TABLE I. Parameters of samples and spin lifetimes for the symmetrically and asymmetrically grown QWs.

\begin{tabular}{lcccccccc}
\hline \hline Sample & $\begin{array}{c}\text { Spacer 1 } \\
l(\mathrm{~nm})\end{array}$ & $\begin{array}{c}\text { QW width } \\
L_{W}(\mathrm{~nm})\end{array}$ & $\begin{array}{c}\text { Spacer } 2 \\
r(\mathrm{~nm})\end{array}$ & $\chi=\frac{l-r}{l+r}$ & $\begin{array}{c}\mu \text { at } 40 \mathrm{~K} \\
\left(10^{5} \mathrm{~cm}^{2} / \mathrm{V} \mathrm{s}\right)\end{array}$ & $\begin{array}{c}n_{s} \text { at } 40 \mathrm{~K} \\
\left(10^{11} \mathrm{~cm}^{-2}\right)\end{array}$ & $\begin{array}{c}\Delta J_{x}^{\mathrm{MPGE}} / \Delta B_{y} \\
(\text { arb. units })\end{array}$ & $\begin{array}{c}\tau_{s} \text { at } 40 \mathrm{~K} \\
(\mathrm{ps})\end{array}$ \\
\hline $\mathrm{A}$ & $\ldots$ & $\ldots$ & 50.0 & $\ldots$ & 1.7 & 1.5 & 18.6 & $\cdots$ \\
$\mathrm{B}$ & 165.0 & 15.0 & 20.0 & 0.78 & 0.98 & 1.6 & 6.6 & $326 \pm 10$ \\
$\mathrm{C}$ & 92.5 & 15.0 & 92.5 & 0.06 & 1.4 & 2.5 & 1.5 & $500 \pm 15$ \\
$\mathrm{D}$ & 20.0 & 15.0 & 165.0 & -0.78 & 2.2 & 3.6 & -2.7 & $192 \pm 9$ \\
$\mathrm{E}$ & 40.0 & 20.0 & 40.0 & 0 & $\cdots$ & $\cdots$ & 0 & $638 \pm 2$ \\
\hline \hline
\end{tabular}

Kerr rotation (TRKR) measurements have been performed. Here, the samples are excited by a circularly polarized laser pulse from a mode-locked Ti:sapphire laser. The laser energy is tuned to excite spin-polarized electron-hole pairs where the electrons are slightly above the Fermi energy of the $2 \mathrm{D}$ electron system. The spin polarization induced in the samples by the pump pulse is probed by a second, timedelayed linearly polarized pulse of the same laser. Because of the magneto-optic Kerr effect, the polarization axis of the reflected probe beam is changed by a small angle, proportional to the $z$ component of the spin polarization in the sample. This Kerr rotation angle is measured by a balanced detector. Standard lock-in technique is used, in
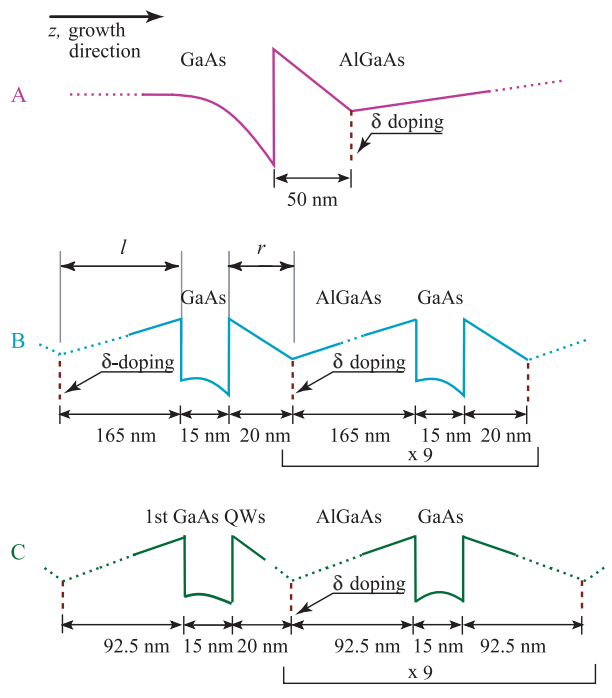

D
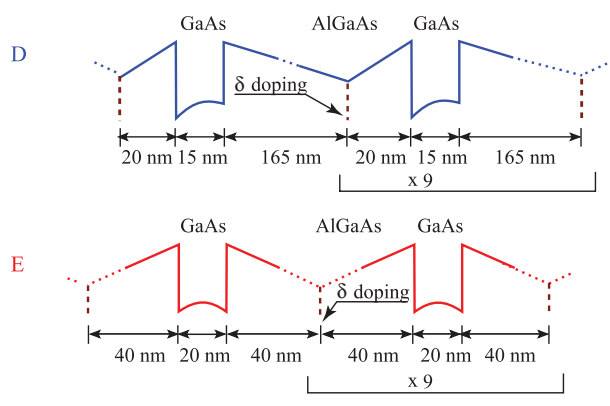

FIG. 1 (color). Band profile of QWs and doping position. which the pump beam is modulated by a flywheel chopper. These measurements are performed at $T=40 \mathrm{~K}$ [12].

The degree of SIA is reflected in the magnetic field dependence of the photocurrent displayed in Fig. 2. To eliminate a magnetic field independent photocurrent offset [13], several times smaller than the photosignal at $B_{y}=$ $\pm 0.5 \mathrm{~T}$, we subtract this background and plot the data in the form $J_{x}^{\mathrm{MPGE}}=J_{x}\left(B_{y}\right)-J_{x}(0)$. The currents shown in Fig. 2 are directly proportional to the applied field, but the slope of $J_{x}^{\mathrm{MPGE}}\left(B_{y}\right)$ is sample dependent. The largest slope is obtained for sample A with the strongest asymmetry while the slope vanishes for the symmetric sample E. In case of sample D, having negative $\chi=(l-r) /(l+r)$, also the slope is negative. The situation changes in the presence of an out-of-plane magnetic field $B_{z}$. For this configuration the MPGE current is observed for all structures including sample $\mathrm{E}$. The result for sample $\mathrm{E}$ is plotted in Fig. 3.

Before continuing, we show within the framework of the phenomenological theory [7] how symmetry is connected with the MPGE effect. As the effect is caused by the lack of an inversion symmetry, both SIA and BIA contribute to the observed phenomena. It turns out that in the case of an inplane external magnetic field the MPGE signal develops only in asymmetric QWs while it is absent in symmetrical QWs having the higher point group symmetry $C_{2 v}$. This effect allows us to analyze the symmetry of the system. Phenomenological theory of the MPGE gives for linearly

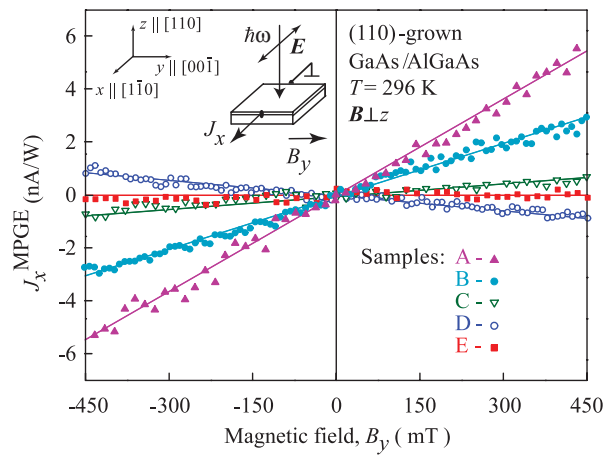

FIG. 2 (color). Magnetic field dependences of $J_{x}^{\mathrm{MPGE}}$ for the radiation polarized along $x$ and an in-plane magnetic field $(\boldsymbol{B} \| y)$. 


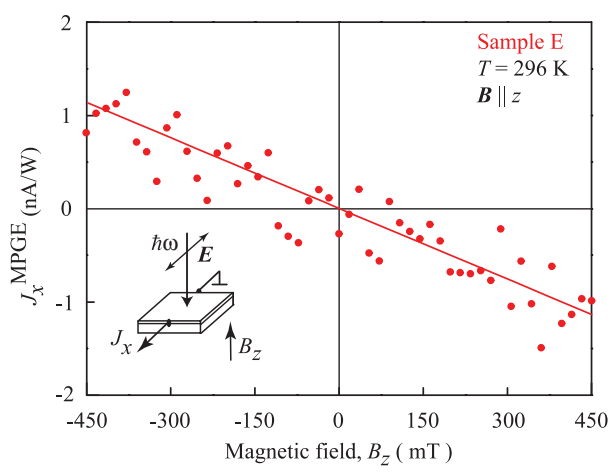

FIG. 3 (color). Magnetic field dependences of $J_{x}^{\mathrm{MPGE}}$ for sample E measured for the radiation polarized along $x$ and a magnetic field perpendicular to the QWs.

polarized radiation at normal incidence [7]

$$
j_{\alpha}^{\mathrm{MPGE}}=\sum_{\beta \gamma \delta} \phi_{\alpha \beta \gamma \delta} B_{\beta} \frac{\left(E_{\gamma} E_{\delta}^{*}+E_{\delta} E_{\gamma}^{*}\right)}{2} .
$$

Here $\phi$ is a fourth rank pseudotensor being symmetric in $\gamma$ and $\delta$ and $B_{\beta}$ and $E_{\gamma}$ are the components of the magnetic field $\boldsymbol{B}$ and radiation electric field $\boldsymbol{E}$, respectively.

We consider MPGE induced by radiation polarized along the $x$ axis, as used in our experiment. For this geometry, the MPGE current $j_{x}^{\mathrm{MPGE}}=\sum_{\beta} \phi_{x \beta x x} B_{\beta}\left|E_{x}\right|^{2}$ is phenomenologically determined by the coupling of the $x$ component of the current polar vector with the axial vector of the magnetic field because $\left|E_{x}\right|^{2}$ is an invariant in (110)-grown structure. Therefore, the photocurrent can occur only for certain $\boldsymbol{B}$ components which are transformed equally to $j_{x}$ for all symmetry operations. While the symmetry of perfectly symmetric (110)-grown QWs belongs to the point group $C_{2 v}$, asymmetric QWs belong to the point group $C_{s}$. The point group $C_{s}$ contains only two symmetry elements: identity and a mirror plane $m_{1}$, perpendicular to the $x$ axis. For $C_{s}$ group symmetry requirements are fulfilled for $j_{x}$ and $B_{y}$ or $B_{z}$ only. Indeed, reflection by the $m_{1}$ plane reverses the sign of $j_{x}\left(j_{x} \rightarrow\right.$ $\left.-j_{x}\right)$ and two components of the magnetic field $\boldsymbol{B}\left(B_{y} \rightarrow\right.$ $-B_{y}$ and $B_{z} \rightarrow-B_{z}$ ). Thus the MPGE can occur for magnetic fields aligned in plane and out of plane of the QW and the photocurrent is given by

$$
j_{x}^{\mathrm{MPGE}, C_{s}}=\phi_{x y x x}^{\mathrm{SIA}} B_{y}\left|E_{x}\right|^{2}+\phi_{x z x x}^{\mathrm{BIA}} B_{z}\left|E_{x}\right|^{2},
$$

with tensors components $\phi_{x y x x}^{\mathrm{SIA}}$ and $\phi_{x z x x}^{\mathrm{BIA}}$ determined by the degree of the SIA and BIA, respectively. If the magnetic field is applied in the $y$ direction, the last term in Eq. (2) becomes zero and the MPGE current is determined solely by the SIA coefficient. The latter determines the degree of SIA and is closely related to our parameter $\chi$.

The experiment displayed in Fig. 2 (samples A to E) shows that the magnitude of the $J\left(B_{y}\right)$ slope strongly depends on the doping profile. Furthermore, if the sign of $\chi$ is reversed (from sample B to D), the slope of the photocurrent gets reversed, too (see Fig. 2). As the MPGE current is proportional to the SIA coefficient, these observations demonstrate that the position of the doping layer can be effectively used for tuning the structure asymmetry strength. In particular, the sign of $\phi_{x y x x}^{\text {SIA }}$ can be inverted by putting the doping layer to the other side of the QW. From this simple $\phi_{x y x x}^{\text {SIA }}$ to $\chi$ relation one would expect that the magnitudes of the $J\left(B_{y}\right)$ slopes of samples B and D are the same. However, the magnitudes of the two slopes differ by about a factor of 2 . This can be attributed to subtle details of impurity incorporation during growth. Indeed, we saw that samples B and D, grown in a similar way but with reversed doping positions, differ by a factor of 2 in electron mobility. Therefore, the structure asymmetry strength of sample B can differ from that of sample D resulting in different slopes. Equation (2) gives also the MPGE current for a magnetic field applied normal to the QW plane, as observed in experiment.

While for an in-plane magnetic field the photogalvanic effect, described by Eq. (1), is observable in asymmetrical structures, it is forbidden in symmetrically grown QWs with the higher point group symmetry $C_{2 v}$. This is caused by the presence of an additional mirror plane, $m_{2}$, being parallel to the QW plane of symmetrically grown (110) structures. Indeed, reflection by this plane does not modify $j_{x}$ but changes the polarity of in-plane axial vector $\boldsymbol{B}$. Therefore, in such systems linear coupling of $j_{x}$ and $B_{y}$ is forbidden. On the other hand, mirror reflection of plane $m_{2}$ does not modify the $z$ component of $\boldsymbol{B}$. Thus the coupling of $j_{x}$ and $B_{z}$ is allowed for reflections by both $m_{1}$ and $m_{2}$ planes, and photocurrent $j_{x}$ can occur in symmetric (110)-oriented QWs in the presence of a magnetic field in the $z$ direction. For this symmetry, Eq. (1) is reduced to

$$
j_{x}^{\mathrm{MPGE}, C_{2 v}}=\phi_{x z x x}^{\mathrm{BIA}} B_{z}\left|E_{x}\right|^{2} .
$$

This equation fully describes the data taken from sample $\mathrm{E}$ (see Figs. 1 and 2). There, no MPGE is observed for inplane magnetic field, but a sizable effect is detected for $\boldsymbol{B}$ applied normal to the QW plane. In the case of sample E, the absence of a magnetic field induced photocurrent in an in-plane $\boldsymbol{B}$ indicates that the QW is highly symmetric and lacks the structure asymmetry. The signal, observed in the same structure for an out-of-plane $B_{z}$ field, stems from the BIA term [see Eq. (3)]. Hence, measurement of the MPGE gives us an experimental handle to analyze the degree of SIA.

The structure inversion asymmetry determines the Rashba spin splitting and therefore controls the D'yakonov-Perel' relaxation [6] for spins aligned along the $z$ direction. Any variation of SIA, e.g., due to asymmetric doping, should result in a variation of the spin relaxation time. To directly demonstrate this connection, we compare spin relaxation rates measured in the symmet- 


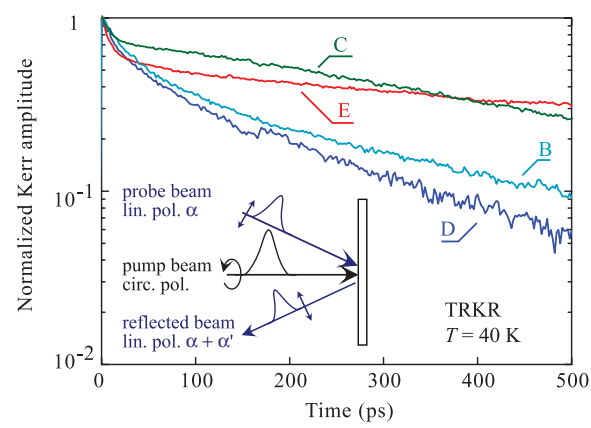

FIG. 4 (color). Kerr rotation measured in asymmetrically grown QWs samples B C, D and symmetrical structure E. We note that the fast decaying components in the TRKR signals at short times are due to spin relaxation of photoexcited holes.

rically doped QW sample $\mathrm{E}$ and the asymmetrically doped QW samples B, C, and D (Fig. 4). We extract the spin lifetime $\tau_{s}$ from TRKR measurements. The time evolution of the Kerr rotation angle tracks the spin polarization within the sample. By fitting an exponential decay function to the data, $\tau_{s}$ is determined. In correspondence to the above photocurrent measurements, indicating a larger degree of asymmetry of the samples B and D compared to C and $\mathrm{E}$, we observe that $\tau_{s}$ in sample $\mathrm{E}$ is more than 3 times larger than that in sample B and about 2 times larger than in sample D (see Table I). We note that in sample $C$ the spin relaxation time is also rather long. In this structure the $\delta$ doping for nine QWs is placed in the center of each barrier separating the QWs and only the first QW is asymmetric. While the photocurrent measurements reflect the asymmetry of the first asymmetric quantum well only, Kerr rotation probes the spin relaxation of the selected QWs, in our case the nine symmetrical ones: Because of the change of the potential profile the asymmetric QW has an effective band gap, which is different from the symmetric QWs and thus does not contribute significantly to the Kerr rotation signal detected at interband resonance conditions with the symmetric QWs.

The relaxation of spins oriented along the $z$ axis due to the D'yakonov-Perel' mechanism in (110)-grown QWs is caused solely by SIA [5]. The measurements on symmetrical sample $\mathrm{E}$ and almost symmetrical sample $\mathrm{C}$ reproduce the results obtained by Ohno et al. [1], where long spin lifetimes were found for nominally undoped (110)-grown QWs by time- and polarization-resolved transmission measurements, as well as those obtained by Döhrmann et al. [3] by means of time- and polarization-resolved photoluminescence (TRPL) on symmetrically grown $n$-doped (110) QWs. We note that in both the TRKR and the TRPL measurements electron-hole pairs are generated in order to probe $\tau_{s}$. Thus, the Bir-Aronov-Pikus mechanism of spin relaxation, in which the randomly fluctuating hole spins couple to the electron spins [14], may not be fully neglected. The difference in $\tau_{s}$ between structures $\mathrm{B}$ and $\mathrm{D}$ having large asymmetry stems from both the different electron mobility and the degree of asymmetry of these samples: In the motional-narrowing regime of D'yakonovPerel' spin relaxation, $\tau_{s}$ is inversely proportional to the momentum relaxation time, which is correlated with mobility.

As an important result of all our measurements we obtained the zero current response and the longest spin relaxation time from the almost symmetrically doped QWs which set an upper limit of spin dephasing in GaAs QWs. This is in contrast to (001)-grown structures where such a geometry results in a substantial SIA [15]. This essential difference stems from the growth temperature, and, subsequently, the diffusion length. Indeed, the growth temperature of high-quality (001)-oriented QWs is higher than $600{ }^{\circ} \mathrm{C}$, while (110) structures are grown at $480^{\circ} \mathrm{C}$ [16]. The high growth temperature of (001)-oriented heterostructures leads to substantial dopant migration and results in structure asymmetry of symmetrically doped QWs. The investigation of MPGE, in particular, the sign inversion by the reversing of structural asymmetry and the zero response of symmetrical structures, provides an effective access to study the symmetry of (110)-oriented QWs. Summarizing, our measurements of the photocurrent give the necessary feedback to reliably grow structures with long spin relaxation times.

We thank E. L. Ivchenko for helpful discussions. This work is supported by the DFG via programs SPP 1285, SFB 689, and GRK 638, Russian President grant for young scientists, and RFBR.

[1] Y. Ohno et al., Phys. Rev. Lett. 83, 4196 (1999).

[2] O.Z. Karimov et al., Phys. Rev. Lett. 91, 246601 (2003).

[3] S. Döhrmann et al., Phys. Rev. Lett. 93, 147405 (2004).

[4] K. C. Hall et al., Appl. Phys. Lett. 86, 202114 (2005).

[5] M. I. D'yakonov and V. Yu. Kachorovskii, Sov. Phys. Semicond. 20, 110 (1986).

[6] M. I. D'yakonov and V. I. Perel', Sov. Phys. Solid State 13, 3023 (1972).

[7] V. V. Bel'kov et al., J. Phys. Condens. Matter 17, 3405 (2005).

[8] S. D. Ganichev and W. Prettl, Intense Terahertz Excitation of Semiconductors (Oxford University, New York, 2006).

[9] E. L. Ivchenko, Optical Spectroscopy of Semiconductor Nanostructures (Alpha Science, Harrow, UK, 2005).

[10] S. D. Ganichev et al., Nature Phys. 2, 609 (2006).

[11] S. D. Ganichev et al., Phys. Rev. B 75, 155317 (2007).

[12] For TRKR we observe a significant reduction of the signal-to-noise ratio as the temperature is increased, making measurements above $100 \mathrm{~K}$ very difficult. Therefore, we choose to use $\tau_{s}$ determined at lower $T$ for comparison.

[13] The background signal is caused by linear photogalvanic effect [9], which is out of the scope of this Letter.

[14] G. L. Bir, A. G. Aronov, and G. E. Pikus, Sov. Phys. JETP 42, 705 (1975).

[15] S. Giglberger et al., Phys. Rev. B 75, 035327 (2007).

[16] L. Pfeiffer et al., Appl. Phys. Lett. 56, 1697 (1990). 